\title{
Fray José Lamana OP (ca.1650-1715), cronista del Real Convento de Predicadores de Zaragoza en la época de los Austrias Menores
}

\author{
Fray José Lamana OP (ca.1650-1715), a historian of the Royal Priory \\ of Preachers of Zaragoza during the Minor Habsburg Age
}

\author{
Alejandro José López Ribao OP \\ ribaotgn@yahoo.es
}

Facultad de Teología San Vicente Ferrer - València

\begin{abstract}
Resumen: Fray José Lamana (Borja ca. 1650 - Zaragoza 1715) fue fraile dominico profeso del Real Convento de Predicadores de Zaragoza. Tras ocupar diversos cargos académicos, especialmente el de rector del Colegio de San Jorge y Santo Domingo de Tortosa, dedicó sus últimos años a ser el bibliotecario y cronista de su convento de filiación. En ese momento redactó dos Lumen Domus o crónicas conventuales de aquel cenobio. La primera se localiza en la Biblioteca de la Universidad de Zaragoza y la segunda, hasta ahora no identificada, en el Archivo Diocesano de la misma ciudad. Ambas permanecen inéditas. El estudio de su figura, su obra como cronista y la importancia de su aportación historiográfica es el objetivo de este artículo.
\end{abstract}

Palabras clave: fray José Lamana, Real Convento de Predicadores de Zaragoza, dominicos, Lumen Domus, crónica conventual

Abstract: Friar José Lamana (Borja ca. 1650 - Zaragoza 1715) was a dominican friar from the Royal Priory of Preachers of Zaragoza. After worked in different academic institutions, especially like rector of the College of San Jorge and Santo Domingo of Tortosa, dedicated his last years to being the librarian and chronicler of his convent. At that time, he wrote two Lumen Domus or convent chronicles. The first is preserved in the University Library of Zaragoza and the second, until now unidentified, in the Diocesan Archive of the same city. Both remain unpublished. The study of his figure, his work as a chronicler and the importance of his historiographical contribution is the main purpose of this article.

Keywords: Father José Lamana, Royal Priory of Preachers, dominicans, Lumen Domus, conventual cronicle 
Alejandro José López Ribao. Fray José Lamana OP (ca. 1650-1715), cronista del Real Convento de Predicadores de Zaragoza en la época de los Austrias Menores

\section{Introducción}

El Real Convento de Predicadores de Zaragoza es el cenobio dominicano perteneciente a la histórica Provincia de Aragón que ha conservado un mayor número de crónicas conventuales manuscritas, así como amplias referencias a los cronistas que las elaboraron. Dichas crónicas, denominadas habitualmente Lumen Domus, y sus autores gozaron de un primer estudio llevado a cabo en los años 1966-1967 por B. Palacios (1966-1967: 183-300). Sin embargo, creemos que aún queda mucho por trabajar, tanto a nivel de su identificación, como de su datación, establecimiento de relaciones intertextuales o incluso incorporación de nuevos manuscritos a su nómina final. Todo ello unido a un estudio más profundo y específico de sus redactores. En este trabajo vamos a centrarnos en uno de ellos, fray José Lamana, dejado en un discreto segundo plano en el referido artículo y olvidado en la actualidad. Con ello queremos no sólo rescatarlo sino mostrar como un buen estudio de estos personajes y su labor enriquece el panorama historiográfico dominicano.

\section{Aproximación biográfica}

Son pocas las noticias biográficas que podemos rastrear sobre fray José Lamana, escrito en algunos casos La Mana por el propio autor. Las fuentes coinciden en que fue originario de Borja, provincia de Zaragoza, e hijo del Real Convento de Predicadores de la capital. En su libro de tomas de hábito se recoge como el «Juebes a 24 de setiembre de 1671 (...) tomaron el hábito para frailes del coro (...) Joseph Lamana de Borja (...) de manos del muy reverendo padre presentado fray Martín Bernad, prior de este convento» (AHPDA 4170: 43r).

Transcurrido el preceptivo año de noviciado profesó el domingo 25 de septiembre de 1672:

\footnotetext{
A las quatro de la tarde en la capilla maior, (...) hicieron proffesión para religiosos del coro los hermanos (...) fray Joseph Lamana de Borja (...) en manos del muy reverendo padre presentado fray Martín Bernad prior de este convento de Predicadores de Çaragoza (...). Y voluntariamente hacían profesión lo firmaron de su mano dichos profesantes (AHPDA 4169: 68r).
}

Trece años más tarde, en 1683, fue elegido lector de artes para el colegio de Santo Domingo y San Jorge de Tortosa. Dicha institución se nutría y regía por frailes venidos de las diversas partes que configuraban la provincia dominicana de Aragón: el principado de Cataluña y los reinos de Aragón y Valencia. Sus estatutos prevenían claramente que nadie pudiese gozar del nombramiento de lector, regente o rector que no hubiese sido por lo menos dos años colegial formal del centro (BC 4709: 204r). De estas prescripciones se deduce que fray José Lamana realizó al menos parte de sus estudios en aquel colegio. Efectivamente, en el libro que registra a los graduados por la universidad se inscribe como bajo el rectorado de fray Tomás Bosch fray José Lamana obtuvo el grado de bachiller en artes el mes de junio del año 1680 (BC 3918: 62r). 
Alejandro José López Ribao. Fray José Lamana OP (ca.1650-1715), cronista del Real Convento de Predicadores de Zaragoza en la época de los Austrias Menores

Para que un fraile pudiese ser colegial era necesario haber sido elegido por su convento de filiación e ir por orden del prior provincial con patente del mismo y la institución conventual. Debía ser examinado por el rector, lectores y regente acerca de su suficiencia en gramática, si venía destinado a los cursos de artes, o de artes, si venía para los de teología (BC 4709: 206v). Tanto los lectores como el rector del centro eran cargos que debían ser proveídos por riguroso turno, es decir, sucesivamente ocupados por un fraile proveniente de Aragón, Cataluña y Valencia. El lector de artes o teología, además de esperar su ocasión, debía haber realizado «opposición delante del muy reverendo padre provincial o en uno de los quatro conventos mayores y esté habilitado en ellos» (Ibid. 210v).

Fray José Lamana a la altura de junio de 1683 cumpliría todos los requisitos académicos. Aunque parece que lo que no era tan claro es si realmente correspondía el turno al lector de Aragón (Ibid. 253). El pleito se resolvió de forma irregular pero salomónica. El capítulo provincial de 1684 decidió zanjar la polémica sobre la nación a la cual correspondía proveer el turno indicando que:

quod finito cursu R.P.Fr. Iosephi Lamana in dicto Collegio actualiter legentis ad Principatum Gotholaniae jus legendi adiucetur et simili modo post cursum dicti Principatus ad Regnum Valentiae inmediatè transeratur et postea ad Regnum Aragoniae (Acta Capituli 1684: 21).

La ordenación se acató y en 1685 fue sustituido como lector de artes por el catalán fray Tomás Bransí.

No tenemos datos demasiado precisos sobre su trayectoria personal y carrera académica entre 1685 y 1702. Sin duda en aquellos años completó sus estudios teológicos y puede ser que fuera cuando llevó a cabo el encargo de studiorum regens en su convento de filiación, oficio recogido en su nota necrológica (AHPDA 4001: 323). También podemos suponer que entre los años 1691 y 1697 volvió a la referida institución dertusense ocupando ahora el cargo de lector en teología, ya que con ese título queda recogido en diversos registros de concesión de grados fechados en aquellos años (BC 3918: 66v-70r).

Lo cierto es que el capítulo provincial celebrado en abril de 1698 decidió instituirlo missionario pro regno aragoniae y promoverlo al título de presentado «pro loco vacante R.P. Praesentati Fr. Iosephi Hyacinti Chia ad Magisterium assumpti» (Acta Capituli 1698: 12 y 17).

Entre 1702 y 1706 ocupó el cargo de rector en el colegio de San Jorge y Santo Domingo de Tortosa. Debido a ello, al desarrollo de este significativo puesto dentro de las instituciones provinciales, son los años en que encontramos más información sobre su persona y en cierta forma sobre su personalidad. El 2 de septiembre de 1702 juró como rector número sesenta y seis del colegio. No había sido el primer candidato de los capitulares, ya que anteriormente habían elegido a fray José Jacinto Chía, el cual «no se numera en los rectores porque no juró por averlo echo antes de venir al colegio provincial de esta provincia de Aragón» (BC 4709: 264). En los escasos cuatro años de su mandato se nos presenta como una persona muy activa, especialmente preocupada por mejorar la situación académica y económica del centro, llevando continuamente a cabo iniciativas hacia aquellos fines y que en ningún caso fueron de despreciar. 
Alejandro José López Ribao. Fray José Lamana OP (ca.1650-1715), cronista del Real Convento de Predicadores de Zaragoza en la época de los Austrias Menores

En junio de 1703 el consejo conventual determinó pedir «la extensión de la universidad para todas facultades». La ocasión parecía propicia al encontrarse en Madrid dos dominicos aragoneses que se valoraban como especialmente dotados para llevar a cabo las gestiones necesarias ante la corte. Uno era fray Luis de Barutell y Erill, socio del Maestro de la Orden, y el otro el lector de prima del colegio fray Lucas Herrero. Ambos «ofrecían hazer mucho y aseguravan les parecía se lograría». Se sumó a la petición la Ciudad y el Cabildo. Sin embargo, el resultado no fue el esperado, ya que «no se logró cosa alguna. Y advierte el maestro Lamana se huviera logrado en tiempo que era confesor de Su Majestad religioso de la orden» (Ibid. 266).

Frustradas las expectativas académicas se decidió emprender una campaña de saneamiento fiscal para incrementar los fondos de la institución, campaña una vez más dirigida contra un interlocutor de peso. En julio de 1703 el consejo resolvió pleitear contra el obispo de Tortosa, don Silvestre García Escolano, para que pagase a la comunidad las pensiones que estipulaban las bulas concedidas a la fundación. Después de varias disputas sobre el valor real del montante se convino que abonara cada año «550 libras de plata valencianas por la pensión de los 300 ducados de oro (...) y por la pensión de los 200 ducados (...) 265 libras» (Ibid. 266). De esta manera empezó a percibir el colegio más de 700 libras anuales.

La necesidad de capital parece justificada por el amplio programa de obras que impulsó. Significativo es que la documentación le dedica un apartado titulado «Memoria de las obras y mejoras del colegio que hizo en su retorado el presentado fray Joseph Lamana desde el septiembre de 1702 (...) hasta los primeros del año 1706». Dicha memoria ocupa un total de tres páginas donde se van describiendo los libros de coro que renovó, las mejoras que introdujo en la biblioteca, tanto en su disposición como en sus fondos bibliográficos, las obras en la pavimentación de la iglesia, sacristía y otros espacios de la casa, así como el saneamiento de las cuentas y rentas de la institución. Elocuentemente la memoria finaliza diciendo: «Otros muchos reparos y mejoras hizo y hubiera hecho mucho más si no hubieran sobrevenido guerras que fue de día 27 de setiembre del año 1705» (Ibid. 268). En total se calculaba que durante su rectorado se gastaron en todas aquellas obras un total de 1.543 libras (Ibid. 211v).

Un detalle a resaltar es que fray José Lamana siempre tuvo un interés especial por la preservación de la memoria y documentación de los conventos a los que estuvo ligado. Ejemplo de ello es la copia que él mismo mandó realizar de los estatutos en las últimas páginas del Lumen Domus del colegio de Tortosa, ya que como indica el narrador «estos estatutos hizo escribir aquí el lector fray Joseph Lamana (...) porque no avía ya sino unos mui derrotados» (Ibid. 211v).

En enero de 1706 daba por terminado su rectorado. La comunidad tuvo problemas para elegir un nuevo rector debido al estado de guerra que se padecía en el Principado, siendo por ello designado como presidente y vicerrector a fray Miguel Vernet desde febrero de 1706 hasta 1708.

El 12 de febrero de 1709 se graduó como maestro en sagrada teología (BUZ 190: 100v). Por desgracia, no sabemos en qué fecha exacta recibió la confirmación de aquel importante título 
Alejandro José López Ribao. Fray José Lamana OP (ca. 1650-1715), cronista del Real Convento de Predicadores de Zaragoza en la época de los Austrias Menores

del que gozó en sus últimos años de vida. Ya que, al no volver a celebrarse capítulo provincial hasta abril de 1717 , fecha en que ya estaba muerto, no es posible encontrar como era usual dicho nombramiento reflejado en las actas capitulares. ${ }^{1}$

Tampoco conocemos la fecha en que volvió a su convento de filiación, pero sí constatamos que a la altura de octubre de 1714 llevaba al menos cuatro años empleados en mejorar la biblioteca conventual suponiéndosele el empleo de bibliotecario (ADZ 197-2: 1r).

Fray José Lamana moría el 15 de noviembre de 1715 y como tal quedaba anotado en el obituario de la comunidad: «Die XV Novembris (...) R.A.P. Magister fr. Josephus Lamana, rector collegii Dertusensis, et hujus conventus studiorum regens. 1715» (AHPDA 4001: 323 y AHPDA 4008). ${ }^{2}$

\section{La obra de fray José Lamana como cronista conventual}

B. Palacios en su repaso a los cronistas del convento aragonés mencionaba un Lumen Domus atribuido a fray José Lamana. De aquel manuscrito, actualmente en la Biblioteca de la Universidad, hacia solo una breve mención. Indicaba que su título completo era Historia de la fundación y sucesos más notables del Convento de Predicadores de Zaragoza, abarcaba cronológicamente desde la fundación hasta el año 1714, había sido brevemente continuado por fray Tomás Madalena, fray Manuel Gallinero y fray Pedro Martínez y que posiblemente su mejor virtud era el haber «refundido y ampliado» el desgraciadamente perdido manuscrito homólogo de fray Raimundo Sáenz, muerto en 1643; manuscrito invariablemente apreciado como «base de todos los posteriores», siendo por ello que tan lamentable pérdida «queda suplida en parte» (B. Palacios 1966-1967: 184 y 188-189). Sin embargo, debemos aclarar que la obra y valoración de fray José Lamana como cronista es bastante más compleja y completa.

En primer lugar, el autor dejó escritos dos Lumen Domus y no uno solo como se afirmaba hasta ahora: un primero conservado en la biblioteca universitaria y un segundo en los fondos del Archivo Diocesano de Zaragoza bajo la signatura de manuscritos 197-2. La atribución queda justificada no sólo por el paralelismo caligráfico sino también por atribuírselo el propio autor y el testimonio de frailes posteriores.

En el verso de la encuadernación del $L D$ I, como denominaremos al conservado en la universidad, una mano posterior consignó:

1 Creemos que el nombramiento se podría encontrar Archivo General de la Orden de Predicadores de Roma signatura IV 176bis, allí hemos podido consultar otras concesiones homologables de la época.

2 Otro problema de que no se celebraran capítulos provinciales durante los años en que duró la Guerra de Sucesión es que las actas del primer capítulo tras ella, el celebrado en Valencia en abril de 1717, no incluyó el usual apartado final titulado Nomina fratrum ac sororum qui ab ultimo capitolo usque ad praesens obierunt y por tanto no se nos consignó ninguna breve nota necrológica del mismo. 
Alejandro José López Ribao. Fray José Lamana OP (ca. 1650-1715), cronista del Real Convento de Predicadores de Zaragoza en la época de los Austrias Menores

\begin{abstract}
Los dos tomos en folio enquadernados en pergamino titulados Historio (o Lumen Domus) del convento escritos por el padre maestro fray Juan Lamana de su misma letra (...) se distinguen en la forma siguiente. El un tomo (...) titula por dentro en su prinicipio así: (In lumen pandit abdita) Lumen Domus o espejo historial del Real Convento de Predicadores de Zaragoza, y sigue diciendo: "en que se trata de su fundación, edificio, yglesia (...)”. Pero este tomo en su seguida de años sólo llega hasta el año 1484 (...). El otro tomo ya tenía por fuerza el título Lumen Domus y no más. Es también de la historia del convento desde su fundación o principio, aunque con distinto método y este tomo lo siguió el maestro Lamana hasta el año 1713.
\end{abstract}

La primera descripción corresponde perfectamente al LD II, conservado en el archivo diocesano, y la segunda al $L D$ I, ambos volúmenes conocidos, apreciados y consignados como obra de fray José Lamana por sus continuadores y compiladores. A continuación, nos disponemos a describir los manuscritos y juzgar su valor como fuentes históricas e historiográficas.

El LD I se titula Historia de la fundación y sucesos más notables del convento de Predicadores de Zaragoza, y el propio autor indicaba que había resumido y refundido en él las noticias que había hallado escritas en el Lumen Domus de fray Raimundo Sáenz. Su objetivo último era el aportar información sobre el convento al lector que no tuviera acceso al archivo conventual, dependencia donde aquel se custodiaba.

Más allá de su declaración de intenciones tenemos que destacar que no sólo es una simple obra de resumen, sino que contiene una importante aportación original. En primer lugar, las noticias consignadas por fray Raimundo Sáenz llegarían como máximo hasta 1643, año de su muerte, y por lo que puede deducirse de la lectura del texto de forma continua hasta 1620 y puntualmente hasta 1639, un priorato posterior al suyo. ${ }^{3}$ Esa cronología indica que los últimos 75 años son obra original de nuestro cronista y si lo valoramos a nivel de extensión corresponde a más de la mitad del manuscrito, en concreto entre los folios 40v y 109v. Aún consideramos mayor la contribución del autor porque el manuscrito va más allá de un simple Lumen Domus conventual, donde se dejaba constancia de los principales acontecimientos comunitarios, englobando otros dos apartados. El primero, que configura una extensa parte, es el enunciado como «Descripción histórica del Real Convento de Predicadores de Zaragoza y sus partes según el estado que han tenido, de su fundación hasta aora, año 1713» (118r-175v). El segundo es el titulado Convento de San Ildefonso, comenzando la narración con una declaración de intenciones: «El padre maestro fray Raimundo Sáenz en el Lumen Domus que escribió trata difusamente del insigne convento de San Ildefonso (...) a folio 427 hasta folio 468 (...). Yo no haré más que un resumen de las cosas más dignas de saberse» (176r).

\footnotetext{
3 BUZ 190: 33r: «Hasta aquí [1620] el Lumen Domus de este convento hablando de este padre maestro Salinas que aun vivía entonces. La tradición es que la santa madre Teresa de Jesús (...) le aseguró de su salvación, encomendándose actualmente a la santa en una estampica suia (como lo representa su imagen y lo dice la inscripción del quadro grande que de este venerable padre ay en el salón de la portería de este convento este año de 1713). Hallo en el libro de los óbitos de los religiosos (...). Aquí rompió el hilo del orden de los priores el padre maestro fray Raymundo Sáenz, y salta al 43 y 44 y da fin [1639]». Hemos transcrito lo referente a fray Francisco Salinas porque nos deja situar el año en que fray Francisco Lamana llevó a cabo la redacción de la noticia.
} 
Alejandro José López Ribao. Fray José Lamana OP (ca.1650-1715), cronista del Real Convento de Predicadores de Zaragoza en la época de los Austrias Menores

Este último apartado ocupa desde el folio 176r hasta el 181v, donde indicará la última fecha de inscripción «Día 28 de julio de 1714 en que concluí esto».

Como se puede apreciar nos hallamos ante un manuscrito en su mayor parte original y volcado en la segunda mitad del siglo XVII y primera década del siglo XVIII, es decir, los años en que se circunscribe la biografía del autor, y por tanto pudiéndose suponer que en su mayoría recoge acontecimientos de los que tuvo constancia de primera mano o que le fueron transmitidos por fuentes directas. De esta manera el Lumen Domus es una fuente de primer orden para escribir la historia de los dominicos en Zaragoza y la provincia de Aragón durante los últimos Austrias y la inmediata Guerra de Sucesión.

Breves ilustraciones de esta afirmación serían al menos tres. La primera la entrada a la ciudad o incluso visita a la casa de personajes ilustres. Es el caso de los monarcas Carlos V, Felipe II, Felipe IV, Carlos II o Felipe V (Ibid. 13r, 21v, 34v, 37r, 45v, 47r, 69v, 70v y 101r-101v). Ejemplo gozoso es la jornada de la reina María Luisa Gabriela de Saboya en abril de 1702, la cual en la fiesta de la Ascensión del Señor acudió al convento

\footnotetext{
oyó misa, después entró en el coro y lo vio y a no ser día de fiesta y ser tarde hubiera entrado en el convento a ver la librería como insinuó la princesa de los Ursinos (...) la que dio palabra volverían una tarde a comer tortillas; pero no tubieron tiempo para esso. En caso que hubieran subido a la librería teníamos intención de presentarle a la reyna unas horicas que ay mui ricas, otras a la princesa, porque ay 2 ricas en el almario de la librería (Ibid. 90v).
}

O la nada digna retirada militar de Felipe V en agosto de 1706, el cual «se vio obligado a retirarse a Zaragoza picándole siempre la retaguardia el señor archiduque con su exército» llegando a la ciudad con su ejército «fatigado del camino, hambriento y mui disminuido a 17 de agosto, un domingo por la mañana, que halló la ciudad sin un pan para socorrer tanta hambre de los soldados» (Ibid. 101r-101v).

Otro ejemplo serían la descripción de las fiestas llevadas a cabo por la canonización de santos propios de la orden y su incorporación a los espacios cultuales de la comunidad. Destacadas serían las de san Pío V, el beato Ceslao y la beata Lucía de Narni en 1713, ya que fray José Lamana fue testigo directo (Ibid. 24v, 26r-27r y 106v-108v).

Por último, no podemos olvidar que en aquellos primeros siglos de la Edad Moderna se sitúan dos de los personajes más importantes para la historia del convento: el cardenal fray Jerónimo Xavierre (1546-1608) y el confesor real e inquisidor general fray Luis de Aliaga (1560-1626). Por ello, el presente manuscrito es una fuente rica en noticias y absolutamente necesaria para conocer su carrera y vínculos con la casa, desde sus donaciones crematísticas hasta sus pompas fúnebres, y desde sus visitas pertinentes hasta la imagen que de sus hijos ilustres quiso preservar la comunidad.

El segundo Lumen Domus tiene características muy diversas. En primer lugar, el arco temporal que abarcan sus 335 páginas es mucho más reducido, se circunscriben entre los años la fundación del 
Alejandro José López Ribao. Fray José Lamana OP (ca.1650-1715), cronista del Real Convento de Predicadores de Zaragoza en la época de los Austrias Menores

convento, hacia 1219, y enero de 1484. Pero que sea más reducido no significa que sea menos interesante, sino que la forma de entender la obra, construirla y el fin para el que fue concebida son muy diferentes. Mientras el LD I se presentaba como un resumen y refundición de los Lumen Domus anteriores el presente cuaderno intenta ir mucho más allá, intenta crear una verdadera historia del convento basándose en todas las fuentes que tuviera el autor a su alcance, contextualizando su discurso y justificando pormenorizadamente sus afirmaciones. No es una simple anotación de eventos sino una narración histórica unitaria y uniformada.

$\mathrm{Al}$ respecto ya es significativo que el manuscrito dé comienzo con un largo prólogo al lector donde deja claras sus intenciones, perfilados sus objetivos y consignada la forma de alcanzarlos. En él explica como empezó la obra para despejarse de sus labores de catalogación como bibliotecario, oficio que venía realizando desde hacía cuatro años, así como para poder «añadir a dicha librería este libro (...) tan deseado de todos como necesario para los hijos deste santo convento» dejándolo así a la consulta común (ADZ 197-2: 1r-1v). A continuación, refiere cuales han sido sus principales fuentes, iniciando por los Lumen Domus que le han hecho sentirse como «un enano (...) sobre los ombros de tantos gigantes». El primero es el de fray Juan de la Cueva, que escribió hacia 1584, contando con documentos que a principios del XVIII ya no se conservaban siendo por ello «más estimando que el oro» su manuscrito. El segundo fue fray Juan de España, el cual «escribió (...) una lista de los priores que ha tenido este convento no desde su fundación sino desde el año 1532 en que comenzó la observancia en esta santa provincia». El trabajo lo prosiguió fray Juan Longo «que lo fue más en virtudes (...) que en el sobrenombre que tenía». El que siguió con el orden de los priores fue fray Pedro Julis llegando hasta el año 1651. Y por último, sobre todos ellos despunta fray Raimundo Sáenz, a quien se le juzga su «inmenso» trabajo hecho con «el amor grande que tenía a esta su casas:

sobre todos estos ha dilatado más pluma en esta materia (...) dividido en dos tomos en folio manuscritos, con mucha erudición histórica. Pero son tantas sus digresiones y no llega lo que nos dexó ordenado de las noticias domésticas sino hasta el año 1276 (Ibid. 1v-2r).

Un último manuscrito apuntado es «los escritos (...) que el (...) padre maestro fray Alonso $\mathrm{H}$ nos dexó (...) a quien celebra (...) con razón el padre maestro fray Raimundo Sáenz». Sin embargo, no sólo quiso basar su historia en fuentes narrativas sino en otras que podríamos entender como económicas:

me he visto precisado a leer más de una vez quantos libros ay en el archivo del gasto y recibo (...) porque como nos falta la historia para ver las cosas de los tiempos antiguos y de la fundación del convento (...) no ay de donde tomar el agua y alguna luz sino de dichos libros que dan algunas noticias (...). He rebuelto y leído quantos papeles, escrituras y libros he podido alcanzar del archivo o fuera de él (Ibid. 2r). 
Alejandro José López Ribao. Fray José Lamana OP (ca.1650-1715), cronista del Real Convento de Predicadores de Zaragoza en la época de los Austrias Menores

Indica además que, como último recurso, ha llegando a hacer uso de las fuentes orales, «muchas veces los ancianos y valiéndome de tradiciones» aunque «solo de las bien fundadas (...) porque muchas he comprobado leyendo ser cuentos de viejas y apócrifos» (Ibid. 2r).

Esta preocupación por la fundamentación de su relato la quiso expresar hasta las últimas consecuencias:

\begin{abstract}
En todo procuraré ajustarme a la verdad según las noticias que hallare. Rara vez me valdré de conjeturas y nunca de discursos que no tienen lugar en la historia sino en lo panegírico, haciendo abultar las cosas tal vez más de lo son. A ninguno doy ventaja en el amor de mi casa, pero grandezas suias mal fundadas no las pondré, porque si bien amicus Plato sed magis amica Veritas (Ibid. 2v).
\end{abstract}

Sin embargo, el corpus documental y aparato crítico de la obra es mucho más extenso y ambicioso. No cuenta sólo con las fuentes enunciadas sino además con un nutridísimo número de obras, en su mayoría publicadas, de las que se va dejando constancia. A modo de ejemplo nos encontramos con hagiografías y primeras crónicas de su orden como la Vida y excelencias de la Madre de Dios de fray Diego Murillo o la vida de santo Domingo de Guzmán de Juan Antonio Flaminio; anales de la religión y de órdenes coetáneas, entre ellos los Annalium Sacri Ordinis Preadicatorum de fray Tomás de Maluenda y la Historia general de la Orden de Nuestra Señora de la Merced Redención de cautivos de fray Alonso Remon; historias de algunas de las provincias destacándose evidentemente la de fray Francisco Diago Historia de la provincia de Aragón de la Orden de Predicadores o la de fray Luís de Sousa Historia de S. Domingos particular do reino e conquistas de Portugal; también historias de los reinos y ciudades donde se sitúa el convento como los Anales de la Corona de Aragón de Jerónimo Zurita y la Historia eclesiástica de la ciudad de Zaragoza desde la venida de Jesucristo, Señor y Redentor nuestro, hasta el año 1575 de Diego de Espés, manuscrito conservado por entonces en la Seo de Zaragoza; y para terminar historias de los monarcas que tuvieron relación con la fundación como De vita et rebus gestis Iacobi primi regis Aragonum cognomento "Expugnatoris" libri XX de Bernardino Gómez Miedes. ${ }^{4}$

Utilizando todas estas obras junto con las crónicas de los «autores domésticos» (Ibid. 4) ${ }^{5}$ y documentos aportados por su investigación fray José Lamana querrá construir una historia crítica y bien enmarcada del convento. Justificando en todo momento, y casi de forma obsesiva, sus afirmaciones. Y enumerando para ello clara y sucesivamente los «pruebas» o «motivos» que le han llevado a cada una de ellas. ${ }^{6}$

4 Es imposible consignar en todas las páginas que se hace referencia a esas obras, así como a muchas otras, pero baste mencionar que el presente listado lo hemos extraído sólo de las ocho primeras.

5 Así llama el propio fray José Lamana a los anteriores cronistas conventuales como por ejemplo se puede leer referido a fray Raimundo Saénz.

6 Ambos términos son los acuñados para el propio autor para enumerar cada una de las autoridades con las que justifica sus afirmaciones (por ejemplo, Ibid. 5-6 y 57).

SCRIPTA, Revista internacional de literatura i cultura medieval i moderna, núm. 16 / desembre 2020 / pp. 163-174 ISSN: 2340-4841 · doi:10.7203/SCRIPTA.16.19225 
Alejandro José López Ribao. Fray José Lamana OP (ca.1650-1715), cronista del Real Convento de Predicadores de Zaragoza en la época de los Austrias Menores

Para finalizar la descripción de este Lumen Domus debemos indicar que el proyecto original se preveía más ambicioso, pareciendo que se quedó simplemente en el primer tomo. La crónica iba a ser una historia del convento en sus cinco centurias de existencia, no sólo las tres primeras. Además, aportaría notica sobre los espacios conventuales, iglesia, capillas y cofradías para acabar nombrando a sus hijos insignes «que en virtud y santidad y en otras líneas ha tenido» con el fin de que el tiempo «no borre del todo la memoria» de forma injusta o por omisión de sus antecesores (Ibid. 2v).

Expuesta brevemente la obra de fray José Lamana podemos concluir que sus dos Lumen Domus son documentos de referencia a la hora de elaborar la historia de la Orden de Predicadores en la provincia de Aragón durante la Edad Moderna. El LD I nos ofrece noticias narradas en su mayor parte por testigos oculares relativas a la vida conventual, religiosa, social y política del periodo. Noticias que no sólo aportan datos históricos sino también la forma de interpretarlos que tuvieron sus protagonistas y la forma que tuvieron de integrarlos en el relato de su historia conventual. El LD II por desgracia no llega hasta los siglos que nos ocupan, pero nos introduce en la forma de hacer y entender un correcto relato histórico de un destacado fraile predicador del momento. Quizás ahora no nos pueda interesar su contenido, pero sí y de manera muy destacada su forma. Nos habla sobre la metodología que evaluó como la más adecuada, sus autores de referencia, el valor de las diversas fuentes que pudo o no pudo consultar, sobre como entendía su oficio y sobre lo que en última instancia debía leerse como una recta historia del cenobio. Nos comparte su visión historiográfica tanto en las palabras del prólogo como en la consecutiva manera de desarrollar los temas. Y es que, como el propio fray José Lamana se definía parafraseando al mismísimo Aristóteles era Amicus Plato sed magis amica Veritas.

\section{Fray José Lamana ¿un cronista olvidado?}

En noviembre de 1715 fray José Lamana moría en su convento de Zaragoza. El obituario de la comunidad no nos aporta gran información sobre su persona o sobre cómo su falta fue sentida por sus coetáneos (AHPDA 4001: 323). Empezaba así un largo periodo en el que deberíamos preguntarnos si su memoria y recuerdo como cronista fue olvidada, minusvalorada o simplemente entendida como innecesaria a tener en cuenta. En nuestra opinión es la última explicación la que debería considerarse como la más adecuada, ya que pareciera que ninguno de los denominados «autores domésticos» tuvo el privilegio de ser recordado como tal.

Preservado, pero no religado, dentro del manuscrito 197-1 del ADZ se conserva un fascículo titulado «Nómina de los religiosos y religiosas que han muerto después de el último capítulo provincial (...) en 9 de maio de 1829» (ADZ 197-1/2: 1r). En él se da noticia de 122 frailes cuyas biografías merecían ser retenidas y releídas como ejemplares para su comunidad. Significativamente, la mitad de los cronistas conventuales que marcó fray José Lamana en el prólogo de su segundo Lumen Domus no aparecen, y los que sí aparecen no son reseñados como tales. Fray Juan Longo, fray Pedro Julis y fray Alonso Bautista no cuentan mención. Fray Juan de España y fray Juan de la Cueva sí 
Alejandro José López Ribao. Fray José Lamana OP (ca.1650-1715), cronista del Real Convento de Predicadores de Zaragoza en la época de los Austrias Menores

merecieron tal honor, aunque en ninguno de los casos se evoca su faceta de cronistas, prefiriéndose reseñar su ardor misionero, celo por la observancia regular, rechazo de cualquier mitra y obvia merecida fama de santidad (Ibid. 9v).

El caso del siempre valorado cronista de referencia fray Raimundo Sáenz es más complejo. De aquel se da breve y diversa noticia: «hijo de este convento, celebre maestro de theología, escritor y historiador crítico» (Ibid. 13v).

En su caso sí remarca su faceta de historiador. ¿'Tan valorado fue siempre su Lumen Domus perdido hasta la fecha? Creemos que la clave es apreciar la diferencia que aquellos frailes de finales del siglo XVIII establecían entre un historiador y un cronista conventual. En el manuscrito de fray José Lamana conservado en el ADZ, anotado justo al margen del juicio que ofrece sobre fray Raimundo Sáenz al comentar que sólo alcanza hasta el año 1276 por sus continuas divagaciones, se puede leer el siguiente añadido por mano posterior: «No tiene razón. Porque dicho padre maestro Sáenz escribió de la centuria de 600 y de muchos prioratos de ella, y de su priorato también»; y por una segunda mano la siguiente interpelación:

\begin{abstract}
Y si por las digresiones lo moteja el maestro La Mana heche la mano a su pecho y vea que se divierte a cosas que no tocan, algo rigurosamente dice el Lumen Domus sobre que el padre maestro Sáenz tuvo el fin y intención de hazer historia universal, y solo que este devemos el poder escribir con alguna certeza y claridad (ADZ 197-2: 2r).
\end{abstract}

De estas afirmaciones se desprenden más preguntas que conclusiones y se nos vuelve a exhortar sobre la necesidad de un nuevo y crítico estudio de las crónicas conventuales conservadas. Sin embargo, no siendo este nuestro objetivo, nos centraremos en lo que parece claro. Se aprecia que fray José Lamana se referiría allí a un manuscrito mucho más ambicioso que un simple Lumen Domus, a un manuscrito de historia universal en el que quedaría englobada la historia conventual. Sería por tanto lícito pensar que fray Raimundo Sáenz además de continuar la crónica conventual incluyendo la de su propio priorato, como es posible leer en posteriores copias, ${ }^{7}$ pudo comenzar otra obra mucho más ambiciosa y alejada de los convencionalismos cronísticos que le valdría el ser recordado e invocado como historiador universal. Categoría ciertamente digna de hacer valer su entrada entre los exclusivos 122 frailes mencionados en el legajo. Los historiadores posteriores quizás debamos comenzar a reflexionar sobre si fray José Lamana también merece ocupar un lugar dentro de ese exclusivo y a nuestro entender demasiado breve listado.

\footnotetext{
7 Nos ha parecido especialmente curioso esa especie de autobiografía que escribió fray Raimundo Sáenz en el Lumen Domus conventual y que podemos leer en la copia posterior del propio fray José Lamana, la cual no deja lugar a dudas sobre su autoría al comenzar «Prior 43 fue el padre maestro fray Raymundo Sáenz, y como escribió el Lumen Domus también escribió su priorato, y de su propria letra dice assí a lo último del $1^{\circ}$ tomo: fue fray Raimundo Sáenz natural de la villa de Autol" extendiéndose a lo largo de cuatro folio» ( BUZ 190: 37v-39r).
} 
Alejandro José López Ribao. Fray José Lamana OP (ca. 1650-1715), cronista del Real Convento de Predicadores de Zaragoza en la época de los Austrias Menores

\section{Fuentes}

Archivo Diocesano de Zaragoza [ADZ] Mss. 197-1/2 y 197-2.

Archivo Histórico Provincia Dominicana de Aragón [AHPDA] Mss. 4001, 4008, 4169 y 4170.

Biblioteca de Catalunya [BC] Mss. 3918 y 4709.

Biblioteca Universidad de Zaragoza [BUZ] Ms. 190.

\section{Bibliografía}

Palacios, B. (1966-1977) «Historiadores y cronistas del Real Convento de Predicadores de Zaragoza», Cuadernos de Historia Jerónimo Zurita, 19-20, pp. 183-300.

(1684) Acta Capituli Provincialis celebrati Caesaragustae in Regio Praedicatorum Conventu die 22 Aprilis 1684, Barcelona.

(1698) Acta Capituli Provincialis celebrati Barcinone in conventu S. Catharinae V. et M. die 19 aprilis anno 1698, Barcelona, 1698.

SCRIPTA, Revista internacional de literatura i cultura medieval i moderna, núm. 16 / desembre 2020 / pp. 163-174 ISSN: 2340-4841 · doi:10.7203/SCRIPTA.16.19225 